\title{
Analysis on relationship between extreme pathways and correlated
} reaction sets

\author{
Yanping $\mathrm{Xi}^{1}$, Yi-Ping Phoebe Chen ${ }^{2}$, Ming $\mathrm{CaO}^{1}$, Weirong Wang ${ }^{3}$ and \\ Fei Wang*1
}

\author{
Address: ${ }^{1}$ School of computer science and technology, Fudan University, Shanghai, PR China, ${ }^{2}$ Faculty of Science and Technology, Deakin \\ University, Melbourne, Australia and ${ }^{3}$ Department of Biochemistry, School of Life Sciences, Fudan University, Shanghai, PR China \\ Email: Yanping Xi -071021055@fudan.edu.cn; Yi-Ping Phoebe Chen - phoebe@deakin.edu.au; Ming Cao - 082024040@fudan.edu.cn; \\ Weirong Wang - wrwang@fudan.edu.cn; Fei Wang* - wangfei@fudan.edu.cn \\ * Corresponding author
}

from The Seventh Asia Pacific Bioinformatics Conference (APBC 2009)

Beijing, China. 13-16 January 2009

Published: 30 January 2009

BMC Bioinformatics 2009, 10(Suppl I):S58 doi:10.1186/I47I-2105-I0-SI-S58

This article is available from: http://www.biomedcentral.com/I47I-2/05/I0/SI/S58

(c) 2009 Xi et al; licensee BioMed Central Ltd.

This is an open access article distributed under the terms of the Creative Commons Attribution License (http://creativecommons.org/licenses/by/2.0), which permits unrestricted use, distribution, and reproduction in any medium, provided the original work is properly cited.

\begin{abstract}
Background: Constraint-based modeling of reconstructed genome-scale metabolic networks has been successfully applied on several microorganisms. In constraint-based modeling, in order to characterize all allowable phenotypes, network-based pathways, such as extreme pathways and elementary flux modes, are defined. However, as the scale of metabolic network rises, the number of extreme pathways and elementary flux modes increases exponentially. Uniform random sampling solves this problem to some extent to study the contents of the available phenotypes. After uniform random sampling, correlated reaction sets can be identified by the dependencies between reactions derived from sample phenotypes. In this paper, we study the relationship between extreme pathways and correlated reaction sets.

Results: Correlated reaction sets are identified for $E$. coli core, red blood cell and Saccharomyces cerevisiae metabolic networks respectively. All extreme pathways are enumerated for the former two metabolic networks. As for Saccharomyces cerevisiae metabolic network, because of the large scale, we get a set of extreme pathways by sampling the whole extreme pathway space. In most cases, an extreme pathway covers a correlated reaction set in an 'all or none' manner, which means either all reactions in a correlated reaction set or none is used by some extreme pathway. In rare cases, besides the 'all or none' manner, a correlated reaction set may be fully covered by combination of a few extreme pathways with related function, which may bring redundancy and flexibility to improve the survivability of a cell. In a word, extreme pathways show strong complementary relationship on usage of reactions in the same correlated reaction set.

Conclusion: Both extreme pathways and correlated reaction sets are derived from the topology information of metabolic networks. The strong relationship between correlated reaction sets and extreme pathways suggests a possible mechanism: as a controllable unit, an extreme pathway is regulated by its corresponding correlated reaction sets, and a correlated reaction set is further regulated by the organism's regulatory network.
\end{abstract}




\section{Background}

In the past decades, genome-scale metabolic networks capable of simulating growth have been reconstructed for about twenty organisms [1]. A framework for constraintbased reconstruction and analysis (COBRA) has been developed to model and simulate the steady states of metabolic networks [2-4]. As reviewed in the literature [5], COBRA has been successfully applied to studying the possible phenotypes. Thus, it has attracted many attentions and gets rapid progress.

The COBRA framework represents a metabolic network as a stoichiometric matrix $\mathrm{S}$. With the homeostatic-steadystate hypothesis and fluxes boundaries, all allowable steady-state flux distributions are limited in a space which can be represented as

$$
\mathbf{S v}=\mathbf{0}, \quad v_{i}^{\min } \leq v_{i} \leq v_{i}^{\max }, \quad i=1, \ldots, n
$$

where $\mathbf{S}_{m \times n}$ is the stoichiometric matrix for a network consisting of $m$ metabolites and $n$ fluxes and $\mathbf{v}_{n \times 1}$ is a vector of the flux levels through each reaction in the system [6].

Given the reversibility of reactions, an internal reversible reaction can be decoupled into two separate reactions for the forward and reverse directions separately. It means all fluxes should take a non-negative value and the solution space is now a convex polyhedral cone in high-dimensional space [6,7]. This convex cone can be spanned by a set of extreme pathways (ExPa), $\left(\mathbf{p}^{i}, i=1, \ldots, k\right)[8,9]$. Every possible steady-state flux distribution in the solution space may therefore be represented as a non-negative combination of extreme pathways $(\mathrm{ExPa})$ :

$$
\mathbf{v}=\sum_{i=1}^{k} \alpha_{i} \mathbf{p}^{i}, \quad \alpha_{i} \geq 0, \quad \forall i
$$

Extreme pathways (ExPa) have the following properties which make them biologically meaningful $[10,11]$ :

1. The ExPa set of a given network is unique.

2. Each ExPa uses least reactions to be a functional unit.

3. The ExPa set is systemically independent which means an ExPa can't be decomposed into a non-negative combination of the remaining ExPas.

A similar network-based pathway definition as ExPa is $\underline{e l e-}$ mentary flux modes (EM) [12-14]. The algorithm for elementary flux modes (EM) treats internal reversible reactions differently from that for ExPas. Actually, ExPa set is a systemically independent subset of elementary flux modes (EM) and each EM can be represented by a non-negative combination of ExPas. The relationship and difference between ExPa and EM have been studied and articulated in literatures $[10,15]$.

ExPas and EMs lead to a systems view of network properties [16] and they also provide a promising way to understand network functionality, robustness as well as regulation $[17,18]$. However, the number of ExPas for a reaction network grows exponentially with network size which makes the use of ExPas for large-scale networks computationally difficult $[19,20]$.

A rapid and scalable method to quantitatively characterize all allowable phenotypes of genome-scale networks is uniform random sampling [21]. It studies the contents of the available phenotypes by sampling the points in the solution space. The set of flux distributions obtained from sampling can be analyzed to measure the pairwise correlation coefficients between all reaction fluxes and can be further used to define correlated reaction sets (CoSet). Correlated reaction sets (CoSet) are unbiased, conditiondependent definitions of modules in metabolic networks in which all the reactions have to be co-utilized in precise stoichiometric ratios [22]. It means the fluxes of the reactions in the same correlated reaction sets (CoSet) go up or down together in fixed ratios. We may think about whether CoSets provide clues about regulated procedures of a metabolic network.

Both ExPas and CoSets are determined by the topology of a metabolic network. Although lots of analyses were done on them separately [23-25], few attention has been paid to the relationship between them. Here, our aim is to reveal the relationship between ExPas and CoSets. We select Escherichia coli core metabolic network, human red blood cell metabolic network and Saccharomyces cerevisiae metabolic network as examples to start our research.

\section{Results and discussion}

\section{Escherichia coli core metabolic network}

We use the E. coli core model published on the web site of UCSD's systems biology research group. It is "a condensed version of the genome-scale E. coli reconstruction and contains central metabolism reactions" [26]. Details of this model can also be found in a published book [27]. The network contains 62 internal reactions, 14 exchange reactions and a biomass objective function.

The computation of the extreme pathways for E. coli core model results in 7784 ExPas, in which 7748 are type I or II ExPas and 36 are type III ExPas (Calculation and classification of ExPas are discussed in Methods section). The type I and II ExPas will be focused on herein and the reason for neglecting type III ExPas will be explained in Methods section. Twenty CoSets are identified on this net- 
Table I: CoSets of E. coli core model.

\begin{tabular}{|c|c|c|}
\hline CoSet ID & CoSet Size & Reactions \\
\hline I & 4 & ACKr, ACt2r, EX_ac(e), PTAr \\
\hline 2 & 3 & G6PDH2r, GND, PGL \\
\hline 3 & 3 & EX_for(e), FORt, PFL \\
\hline 4 & 3 & D_LACt2, EX_lac_D(e), LDH_D \\
\hline 5 & 3 & CYTBD, EX_o2(e), O2t \\
\hline 6 & 3 & ADHEr, ETOHt2r, EX_etoh(e) \\
\hline 7 & 2 & TALA, TKTI \\
\hline 8 & 2 & ICL, MALS \\
\hline 9 & 2 & GAPD, PGK \\
\hline 10 & 2 & FUM, SUCD4 \\
\hline II & 2 & FBA, TPI \\
\hline 12 & 2 & EX_Pyr(e), PYRt2r \\
\hline 13 & 2 & EX_h2o(e), $\mathrm{H} 2 \mathrm{Ot}$ \\
\hline 14 & 2 & EX_glc(e), GLCpts \\
\hline 15 & 2 & ENO, PGM \\
\hline 16 & 2 & $\mathrm{CO} 2 \mathrm{t}, \mathrm{EX} \_\mathrm{co} 2(\mathrm{e})$ \\
\hline 17 & 2 & AKGt2r, EX_akg(e) \\
\hline 18 & 2 & AKGDH, SUCOAS \\
\hline 19 & 2 & ADKI, PPS \\
\hline 20 & 2 & ACONT, CS \\
\hline
\end{tabular}

This table lists all CoSets of E. coli core model. We give each CoSet an ID and list it in the First column. We list CoSet size and reactions it contained in the second and third column. Reaction names are in abbreviated form. The abbreviation list is in table 7 and additional file I.

work based on pairwise correlation coefficients between all reaction fluxes and listed in table 1.

For each CoSet $\mathbf{C}_{j}$, we check how many type I and II ExPas use $k$ reactions in $\mathbf{C}_{j}$, where $k$ ranges from zero to the size of $\mathbf{C}_{j}$. The result is shown in table 2. Taking CoSet 3 as an example, from table 1 and 2, we can find that 3 reactions ('EX_for(e), FORt, PFL') belong to CoSet 3. Among all the type I and II ExPas, 5026 of them use all of these 3 reactions and 2722 use none of them. No ExPa uses one or two reactions. It is clear that each ExPa of E. coli core model covers in each CoSet in an 'all or none' manner. We also calculate, for each ExPa $\mathbf{p}^{i}$, the ratio of reactions in any CoSet which is fully covered by $\mathbf{p}^{i}$ to all reactions in $\mathbf{p}^{i}$. The distribution of the ratios is shown in Figure 1. Each ExPa of E. coli core model covers at least one CoSet. The coverage rates are higher than $40 \%$ which implies that ExPas are under well control of CoSets.

\section{Human red blood cell metabolic network}

Human red blood cell (RBC) metabolic network has been well reconstructed and simulated [28-31]. The RBC model consists 39 metabolites, 32 internal metabolic reactions (See additional file 2) as well as 19 exchange fluxes (Figure 2) [25].

There are 55 ExPas calculated from the stoichiometric matrix of RBC model, among which 39 are type I or II ExPas and the others are type III ExPas. We focus on type I and II ExPas only. Type I and II ExPas are described in additional file 2. Eight CoSets are identified on RBC model. All CoSets are listed in table 3. The CoSets of RBC show agreement with the currently known regulatory structure [32]. There are 12 reactions regulated by inhibitors and activators or through post-translational modification. Most of them belong to some CoSet and most of CoSets have at least 1 regulated reaction. For example, regulated reactions 'G6PDH' and 'PDGH' belong to CoSet 1; 'TKI', 'TA' and 'TKII' belong to CoSet 2; 'RPI' and 'PFK' belong to CoSet 3; 'EN' and 'PK' belong to CoSet 4; 'AdPRT' belongs to CoSet 7 . Although there's no regulated reaction in CoSet 6 , it shares the metabolite ' $\mathrm{R} 5 \mathrm{P}^{\mathrm{\prime}}$ with regulated reactions 'R5PI', 'TKI' and 'PRPPsyn'. So the reactions in CoSet 6 can be considered as being regulated indirectly. The other 2 reactions, 'PRPPsyn' and 'IMPase', don't belong to any CoSet.

The relationship between ExPas and CoSets is shown in table 4. Each CoSet is covered by an ExPa in an 'all or none' manner, except the CoSets 1 and 3 . As for CoSets 1 and 3, some ExPas cover them in an 'all or none' manner and others cover them in 'one or all but one' mode. We look over the two exceptions to see which reactions are used by each ExPa and which are not used. As to CoSet 1 , there are 24 ExPas covering it in an 'all or none' manner and 15 ExPas overlapping with it in a 'one or all but one' mode. Among these 15 ExPas, 6 ExPas use one and the same one reaction 'PGI' while other 9 ExPas use all the reactions in CoSet 1 only except the reaction 'PGI'. Similar situation can be found in CoSet 3. There are 12 ExPas overlapping with it in a 'one or all but one' mode, among which 6 ExPas use the same reaction 'R5PI' while other 6 ExPas cover all reactions but 'R5PI'.

The reasons for the complementary relationship on usage of reactions in CoSet 1 and CoSet 3 are as follows. 'PGI' 
Table 2: Relationship between ExPas and CoSets for E. coli core metabolic network.

\begin{tabular}{|c|c|c|c|c|c|c|}
\hline \multirow[t]{2}{*}{ CoSet ID } & \multirow[t]{2}{*}{ CoSet Size } & \multicolumn{5}{|c|}{ Number of ExPas using $\mathrm{k}$ reactions of a CoSet } \\
\hline & & 0 & 1 & 2 & 3 & 4 \\
\hline I & 4 & 6652 & 0 & 0 & 0 & 1096 \\
\hline 2 & 3 & 3556 & 0 & 0 & 4192 & - \\
\hline 3 & 3 & 2722 & 0 & 0 & 5026 & - \\
\hline 4 & 3 & 7151 & 0 & 0 & 597 & - \\
\hline 5 & 3 & 1306 & 0 & 0 & 6442 & - \\
\hline 6 & 3 & 3984 & 0 & 0 & 3764 & - \\
\hline 7 & 2 & 3556 & 0 & 4192 & - & - \\
\hline 8 & 2 & 5223 & 0 & 2525 & - & - \\
\hline 9 & 2 & 928 & 0 & 6820 & - & - \\
\hline 10 & 2 & 2240 & 0 & 5508 & - & - \\
\hline 11 & 2 & 1352 & 0 & 6396 & - & - \\
\hline 12 & 2 & 7106 & 0 & 642 & - & - \\
\hline 13 & 2 & 1983 & 0 & 5765 & - & - \\
\hline 14 & 2 & 904 & 0 & 6844 & - & - \\
\hline 15 & 2 & 928 & 0 & 6820 & - & - \\
\hline 16 & 2 & 1697 & 0 & 6051 & - & - \\
\hline 17 & 2 & 6499 & 0 & 1249 & - & - \\
\hline 18 & 2 & $567 \mid$ & 0 & 2077 & - & - \\
\hline 19 & 2 & 5181 & 0 & 2567 & - & - \\
\hline 20 & 2 & 2193 & 0 & 5555 & - & - \\
\hline
\end{tabular}

This table illustrates relationship between ExPas and CoSets for E. coli core metabolic network. For each CoSet, we calculate how many ExPas cover $k$ reactions in it where $k$ ranges from 0 to size of this CoSet.

belongs to one of 'historical' metabolic pathways named Embden-Meyerhof-Parnas pathway (EMP), while all other internal reactions in CoSet 1 are in pathway Pentose Phosphate Pathway (PPP). As for CoSet 3, 'R5PI' belongs to pathway PPP and all other reactions are in EMP. Since EMP provides the metabolite 'G6P' to PPP and inversely, PPP offers the metabolite 'GA3P' to EMP, the two pathways should cooperate with each other to fulfill the functions of the metabolic network. In order to work normally, the metabolic network may either utilize an ExPa using all the reactions in CoSet 1 (CoSet 3) or com- bine two (or more) ExPas together to fully cover CoSet1 (CoSet 3). By splitting some CoSet on different ExPas, it may bring redundancy and flexibility which are important properties for a cell to survive in various environments.

Both 'Ex_NADP' and 'Ex_NADPH' belong to CoSet 1, indicating the need of RBC cell to balance the NADPH/ NADP ratio. According to "historically" partition of metabolic pathways, when pathway PPP is up-regulated, the quantity of NADP increases. When metabolic pathway EMP is up-regulated, the quantity of NADPH comes up. 


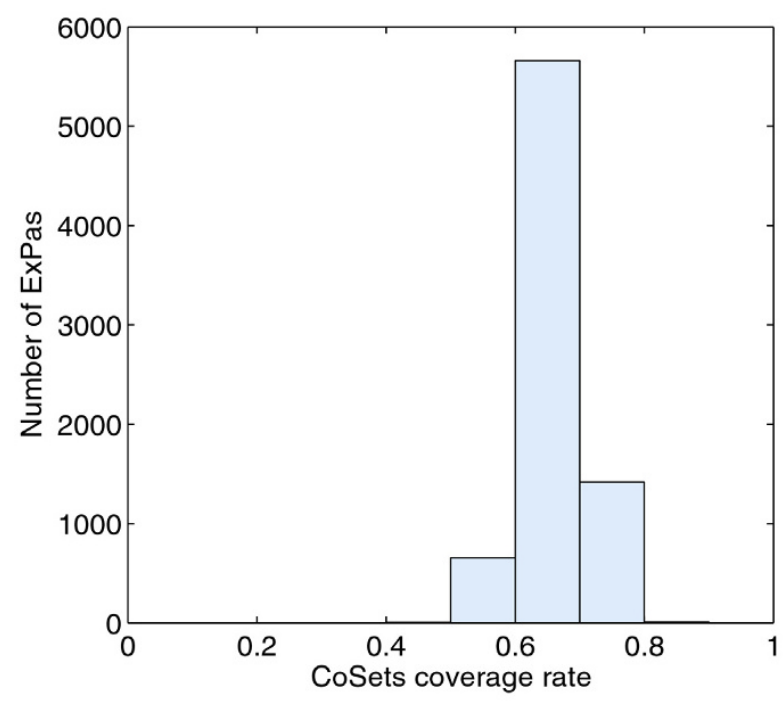

Figure I

CoSets coverage rate of ExPas of E. coli core metabolic network. The $y$-axis indicates the number of extreme pathways which have the corresponding CoSets coverage rates; the $x$-axis lists the Cosets coverage rates, ranging from 0 to $\mathrm{I}$.

From the point of view of ExPa, 'Ex_NADP', 'Ex_NADPH' are used together in opposite direction by ExPas. It means that the fluxes through these reactions increase or decrease together. As a result, the quantity of NADP increases when that of NADPH decreases and vice versa. Situation is similar for reactions 'Ex_NAD' and 'Ex_NADH' in CoSet 5.

Figure 3 is the CoSets coverage rate of RBC model. Though the coverage rates are not as high as of those of $E$. coli core metabolic network, nearly $1 / 3$ ExPas of RBC model has a CoSets coverage rate higher than $20 \%$. There are 7 ExPas whose CoSets coverage rate is 0 . All these 7 ExPas utilize relatively few reactions (1-3 internal reactions as well as the corresponding exchange reactions), among which, ExPas 10 and 11 utilize the regulated reaction 'IMPase', ExPas 12 and 13 are type II ExPas which serve to dissipate excess ATP, and ExPas 14, 15, 16 which participate in nucleotide metabolism may be regulated by the quantity of inosine and adenosine. In short, ExPas are in control of the regulatory structure of the metabolic network and our study suggests that the regulatory command usually spread from the regulated reactions to CoSets and finally to the related ExPas.

\section{Saccharomyces cerevisiae metabolic network}

A full compartmentalized genome-scale metabolic model for S. cerevisiae, iND750, has been reconstructed and validated in 2004 [33]. We use this model to represent the metabolism of S. cerevisiae. Model iND750 accounts for
646 metabolites, 1149 internal reactions as well as 116 exchange fluxes excluding the objective reaction. Since the scale of iND750 is too large, enumerating all the ExPas of the model is computational intractable. Thus we samples a subset of ExPas to represent the whole ExPas (See Methods Section). The sampling procedure has executed 1000 times with 250-300 internal reactions being randomly removed out every time and finally resulted a sample set of 56496 unique ExPas. The lengths of sample ExPas range from 20 to 80 (Figure 4). It is difficult to sample the ExPas containing more than 80 reactions within acceptable cost of time.

One hundred and thirty five CoSets have been identified for this model. Some CoSets, especially the CoSets containing more than 5 reactions, have no sample ExPa passing through as if they are forgotten by the metabolic network. We name them CoSets of solitary island. We have tried different methods, such as removing all reactions which cannot be reached from a certain CoSet of solitary island, to sample some ExPas passing through the 'solitary island' but in vain because the sampling procedures take too much time. It seems that, the reactions in a CoSet of solitary island together with the reactions related to them form a complex network, and ExPas usually have to take a long way to go from some exchange reactions to a CoSet of solitary island and finally reach other exchange reactions. Because of the network's complexity, there are many bypaths along the road which causes a combinatorial explosion. So a CoSet of solitary island is not really solitary, and it is not too few but too many ExPas passing through these CoSets that prevent the ExPas computation algorithm, one step of which is enumerating all possible combinatorial paths, from catching them.

CoSets and the relationship between ExPas and CoSets are completely listed in additional files 4 and 5 separately. Due to the limited space, part of them are shown in table 5 and table 6 . Figure 5 is the CoSets coverage rate distribution of S. cerevisiae model. We find that leaving out of the CoSets of solitary island, almost all the CoSets are covered by ExPas in an 'all or none' manner except CoSet 30 which is covered by ExPas in a complemental mode. CoSet 30 has three reaction members, 'AKGMAL', 'AKGt2 $r$ ' and 'MALt2r'. Reaction 'AKGMAL' transports alpha ketoglutarate (AKG) and malate (MAL) across the epicyte in opposite directions. Reaction 'AKGt2r' transports AKG and hydrogen ion $(\mathrm{H})$ across the epicyte in the same directions. And 'MALt2r' transports MAL and $\mathrm{H}$ across the epicyte in the same directions as well. If the quantity of AKG rises, the fluxes through 'AKGMAL' will grow up taking AKG and $\mathrm{H}$ out of the cell and bringing MAL into the cell. As a result, the quantity of $\mathrm{H}$ rises causing an increase on the flux of 'MALt2r'. Vice versa. These three reactions work together to balance the AKG/MAL ratio inside the cell and 


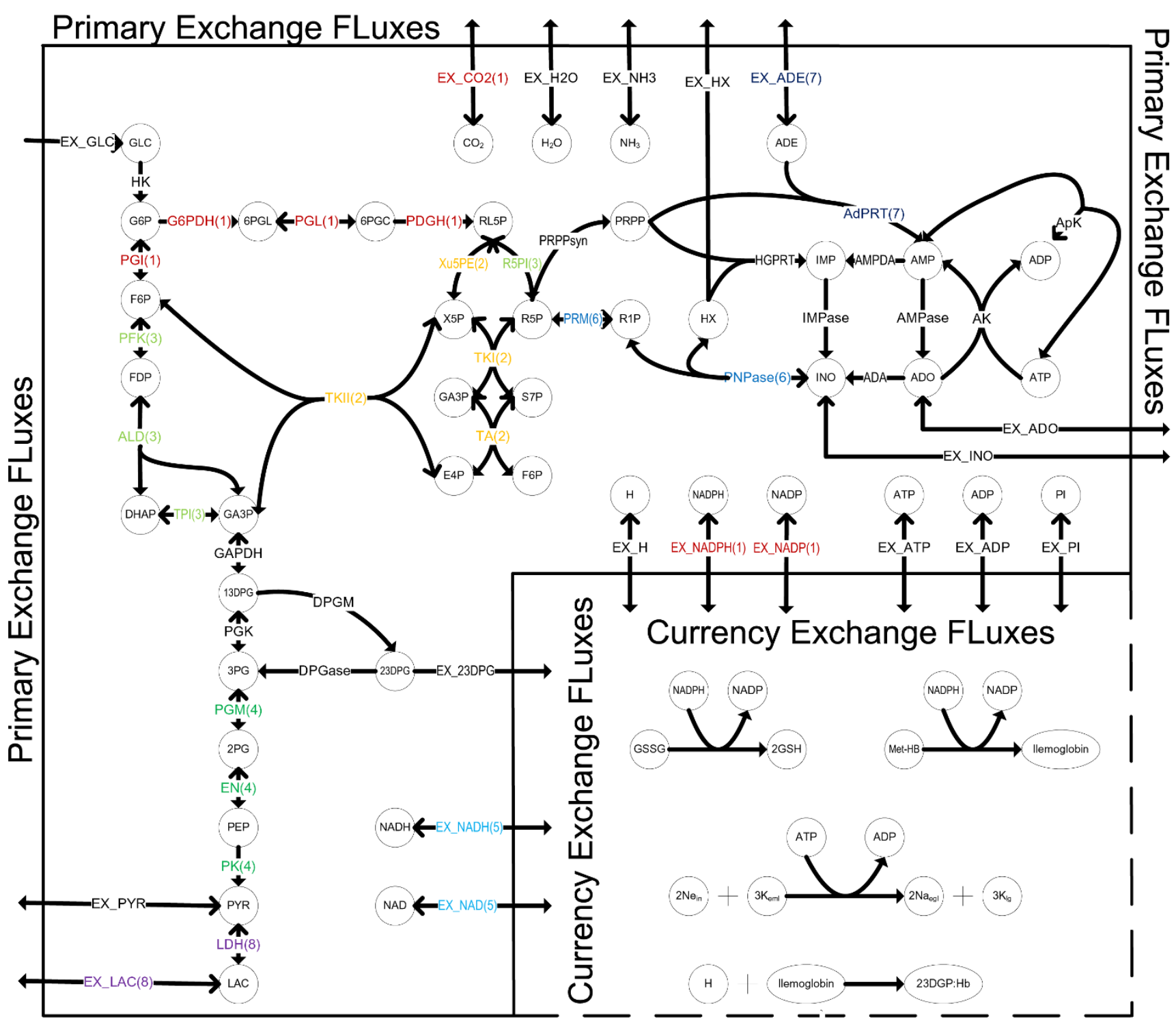

Figure 2

Metabolic maps of RBC. The graph is adapted from [25]. CoSet label of each reaction is added and different symbols are used to represent forward $(\rightarrow)$ and reverse $(\rightarrow)$ directions separately.

thus form a CoSet. Among the sample ExPas, we find that some of them utilize 'AKGMAL' and 'AKGt2r' while others use 'MALt2r' only. But, there are also some ExPas utilizing 'AKGt2r' while we don't find any sample ExPas that use the other two reactions in the CoSet. However, according to the above analysis, there should be some complemental ExPas utilizing reactions in the CoSet other than 'AKGt2r'. Otherwise, the cell will die due to the insupportable internal environment. Since the whole ExPa set is extremely large, the available ExPa sample set can only give a glance at the tremendous ExPa set and will certainly lose some information.
The scale of S. cerevisia metabolic network is much larger. However, complementary relationship on usage of reactions in a CoSet is repeated as that in E. coli core metabolic network and RBC metabolic network.

\section{Conclusion}

In this study, we investigated the relationship between CoSets and ExPas on the in-silicon representations of three metabolic networks. These models are different in species and scale. However, the experiment on each model leads to similar results that ExPas show strong complementary relationship on the usage of reactions in the same CoSet. It implies that this kind of relationship may exist in most 
Table 3: CoSets of RBC metabolic network.

\begin{tabular}{ccc}
\hline CoSet ID & CoSets Size & Reactions \\
\hline 1 & 7 & PDGH, Ex_CO2, Ex_NADPH, PGI, PGL, G6PDH, Ex_NADP \\
\hline 2 & 4 & Xu5PE, TKI, TKII, TA \\
\hline 3 & 4 & PFK, ALD, TPI, R5PI \\
\hline 5 & 3 & PGM, EN, PK \\
\hline 6 & 2 & Ex_NAD, Ex_NADH \\
\hline 7 & 2 & PNPase, PRM \\
\hline 8 & 2 & AdPRT, Ex_ADE \\
\hline
\end{tabular}

This table lists all CoSets of RBC model. We give each CoSet an ID and list it in the First column. We list CoSet size and reactions it contained in the second and third column. Reaction names are in abbreviated form. The abbreviation list is in table 7 and the list of internal reactions is in additional file 2 .

of organisms. Since both CoSets and ExPas are generated from the topology information of metabolic networks, this phenomenon may reflect some inherent properties resulting from the topology constraints composed on the networks.

Moreover, our study not only reveals the interesting relationship between CoSets and ExPas but also provides a new sight of how the metabolic network works and how it is adjusted. The strong relationship between CoSets and ExPas provides clues about regulated procedure of a metabolic network, thus suggests a possible mechanism of how a metabolic network transferring its phenotype from one steady state to another. As functional units, ExPas are in control of the regulatory structure of the metabolic network, and the regulatory command usually spreads from regulated reactions to CoSets and finally to the related ExPas. As fluxes through each ExPa change according to the regulatory orders from its corresponding CoSets, the flux distribution of the whole metabolic network transfers towards a new steady state. By interrogating the relationship between CoSets and ExPas, we can tell which ExPas are possible to be up (down) regulated caused by an increasing (decreasing) flux in a given CoSet. This result

Table 4: Relationship between ExPas and CoSets for RBC metabolic network.

\begin{tabular}{|c|c|c|c|c|c|c|c|c|c|}
\hline \multirow[t]{2}{*}{ CoSet ID } & \multirow[t]{2}{*}{ CoSets Size } & \multicolumn{8}{|c|}{ Number of ExPas using $\mathrm{k}$ reactions of a CoSet } \\
\hline & & 0 & 1 & 2 & 3 & 4 & 5 & 6 & 7 \\
\hline I & 7 & 18 & 6 & 0 & 0 & 0 & 0 & 9 & 6 \\
\hline 2 & 4 & 21 & 0 & 0 & 0 & 18 & - & - & - \\
\hline 3 & 4 & 18 & 6 & 0 & 6 & 9 & - & - & - \\
\hline 4 & 3 & 27 & 0 & 0 & 12 & - & - & - & - \\
\hline 5 & 2 & 19 & 0 & 20 & - & - & - & - & - \\
\hline 6 & 2 & 24 & 0 & 15 & - & - & - & - & - \\
\hline 7 & 2 & 30 & 0 & 9 & - & - & - & - & - \\
\hline 8 & 2 & 37 & 0 & 2 & - & - & - & - & - \\
\hline
\end{tabular}

This table illustrates relationship between ExPas and CoSets for RBC metabolic network. For each CoSet, we calculate how many ExPas cover $k$ reactions in it where $k$ ranges from 0 to size of this CoSet. 


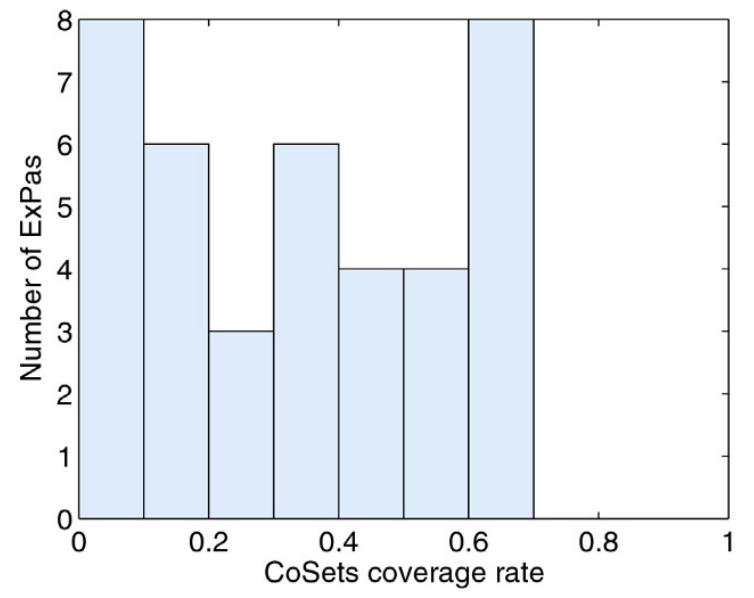

Figure 3

CoSets coverage rate of ExPas of RBC metabolic network. The $y$-axis indicates the number of ExPas which have the corresponding CoSets coverage rates; the $x$-axis represents the Cosets coverage rates, ranging from 0 to $\mathrm{I}$.

may help predict the function of regulatory factors acting on metabolism. However, in order to answer the question which ExPas are really regulated, more information should be considered, such as regulatory structure of the

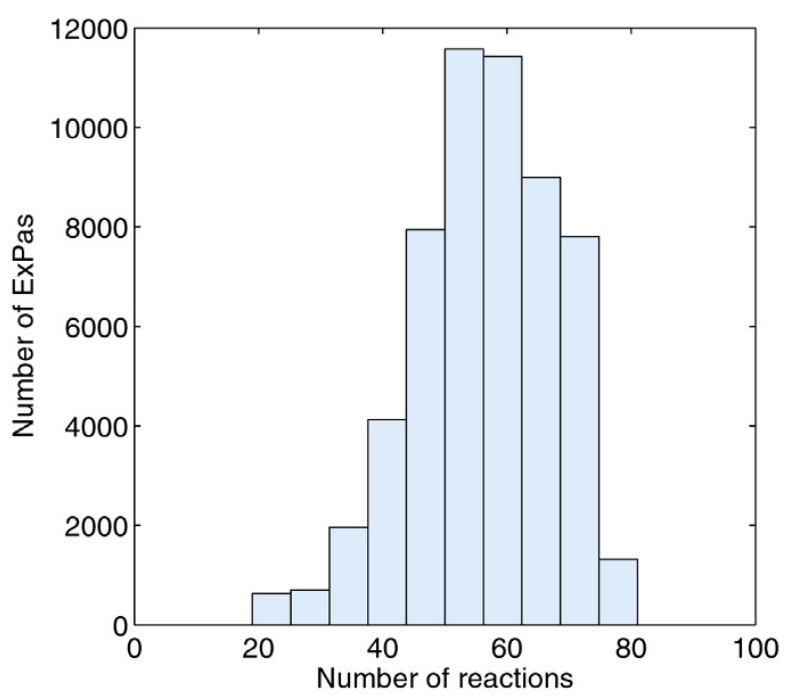

\section{Figure 4}

Length of iND750's sample ExPas. The y-axis indicates the number of ExPas which consist of the corresponding number of reactions; the $x$-axis represents the number of reactions contained in a single ExPa. The ExPa sampling process found no ExPa whose length is less than 20 or more than 80.

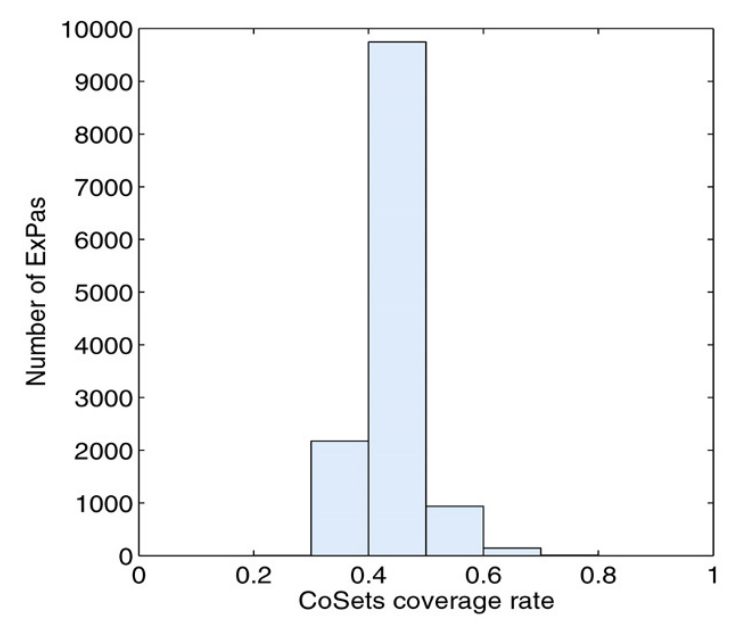

Figure 5

CoSets coverage rate of ExPas of S. cerevisia model. The $y$-axis indicates the number of extreme pathways which have the corresponding CoSets coverage rates; the $x$-axis lists the Cosets coverage rates, ranging from 0 to $\mathrm{I}$.

metabolic networks as well as kinetic and thermodynamic constraints, which will be our future work.

\section{Methods}

\section{ExPas computation and classification}

ExPas are computed by an open source tool, 'expa', developed by Steven L. Bell and Bernhard O. Palsson [34]. The exchange fluxes can be separated into two groups: primary exchange fluxes and currency exchange fluxes. Primary exchange fluxes are external fluxes and currency exchange fluxes are fluxes external to metabolism but internal to the cell [27]. ExPas can be divided into three categories according to their use of exchange fluxes [35]. Type I ExPas utilize primary exchange fluxes as well as currency exchange fluxes. Type II ExPas involve currency exchange fluxes only. Type III ExPas are solely internal cycles without any exchange fluxes. Since type III ExPas are thermodynamically infeasible [36], we neglect type III ExPas and only focus on those of type I and II.

\section{CoSets computation}

The CoSets of each metabolic model is generated by COBRA toolbox, an integrated toolbox of functions which are useful for analysis and simulation of organism's metabolic behavior [22]. For each model, uniform random sampling has been done first in the condition of optimum growth and results in 100,000 unique sample flux distributions that are available to the network. Then, 10,000 samples have been randomly selected and used to measure the pairwise correlation coefficients between reactions. We set the threshold of square pairwise correlation 
Table 5: CoSets of S. cerevisiae metabolic network.

\begin{tabular}{|c|c|c|}
\hline CoSet ID & CoSet Size & Reactions \\
\hline II & 5 & HETZK, HMPKI, PMPK, TMN, TMPPP \\
\hline 13 & 5 & ACGKm, ACOTAim, AGPRim, ORNTACim, ORNt3m \\
\hline 20 & 3 & PGCD, PSERT, PSP_L \\
\hline 22 & 3 & GCCam, GCCbim, GCCcm \\
\hline 25 & 3 & CYTK2, DCTPD, NDPK7 \\
\hline 27 & 3 & CYOOm, CYOR_u6m, O2tm \\
\hline 29 & 3 & ARGSL, ARGSSr, OCBTi \\
\hline 30 & 3 & AKGMAL, AKGt2r, MALt2r \\
\hline 31 & 3 & AKGDam, AKGDbm, SUCOASm \\
\hline 33 & 3 & ACSm, ADKIm, PPAm \\
\hline 34 & 3 & ACLSm, DHADIm, KARAIim \\
\hline 35 & 3 & ABTA, GLUDC, SSALy \\
\hline 38 & 3 & 34HPPt2m, TYRTAm, TYRt2m \\
\hline
\end{tabular}

This table lists the no solitary island CoSets of $S$. cerevisiae metabolic network model with set size no less than 3 . We give each CoSet an ID and list it in the First column. We list CoSet size and reactions it contained in the second and third column. Reaction names are in abbreviated form. The abbreviation list is in table 7 and additional file 3 .

coefficient to $1-1 e^{-8}$ while identifying CoSets of each metabolic network assuring that reactions in the same CoSets have strong correlation with each other. The procedure of CoSets identification has been carried out 20 times for each model and the results are quite stable.

\section{Sampling for ExPa subset}

We randomly delete a few reactions in $S$. cerevisiae's iND750 model, and enumerate all the ExPas of the sub network. Then, the dimensions of deleted reactions are inserted back with zeros to these ExPas. As proved in Theorem 1, the ExPa set derived from sampling is a subset of the whole ExPa set of iND750. One thousand ExPa sets of different sub networks of iND750 model have been generated and merged together without redundancy. The union of all these ExPas constitute the sample set of ExPas used in the analysis on Saccharomyces cerevisiae metabolic network.

Theorem 1. Suppose $G$ is a metabolic network and $\mathbb{P}$ is the ExPa set of $G$, then for any sub network $G^{\prime}$, its ExPa set $\mathbb{P}^{\prime}$ is a subset of $\mathbb{P}$.
Proof. We assume that the available steady state flux distribution ( $\mathbf{v}$ ) of $G$ lies in the convex cone $c$ :

$$
\mathbf{S v}=\mathbf{0}, v_{i} \geq 0, i=1, \ldots, n
$$

Without loss of generality, we assume $G^{\prime}$ is generated from $G$ by deleting reactions $v_{k}, v_{k+1}, \ldots, v_{n}$, then the steady state flux distribution of $G^{\prime}$ lies in the convex cone $c^{\prime}$ :

$$
\mathbf{S v}^{\prime}=\mathbf{0}, \quad \begin{cases}v_{i}^{\prime} \geq 0, & i=1, \ldots, k-1 \\ v_{i}^{\prime}=0, & i=k, \ldots, n\end{cases}
$$

Assuming that $\mathbb{A}=\left\{\mathbf{a}^{i} \mid \mathbf{a}^{i} \in \mathrm{C}\right.$ and $\left.a_{j}^{i}=0, j=k, \ldots, n\right\}$. Obviously, $\mathbb{A}=\mathrm{C}^{\prime}$.

$\forall \mathbf{a}^{i} \in \mathbb{A}, \exists \mathbb{P}^{\prime \prime} \subseteq \mathbb{P}$, that

$$
\mathbf{a}^{i}=\sum_{i=1}^{\left|\mathbb{P}^{\prime \prime}\right|} \alpha_{i} \mathbf{p}^{i}, \quad \alpha_{i} \geq 0, \quad \forall i
$$


Table 6: Relationship between ExPas and CoSets for S. cerevisiae model.

\begin{tabular}{|c|c|c|c|c|c|c|c|}
\hline \multirow[t]{2}{*}{ CoSet ID } & \multirow[t]{2}{*}{ CoSet Size } & \multicolumn{6}{|c|}{ Number of ExPas using $\mathrm{k}$ reactions of a CoSet } \\
\hline & & 0 & 1 & 2 & 3 & 4 & 5 \\
\hline 11 & 5 & 56445 & 0 & 0 & 0 & 0 & 51 \\
\hline 13 & 5 & 49250 & 0 & 0 & 0 & 0 & 7246 \\
\hline 20 & 3 & 39967 & 0 & 0 & 16529 & - & - \\
\hline 22 & 3 & 54670 & 0 & 0 & 1826 & - & - \\
\hline 25 & 3 & 56393 & 0 & 0 & 103 & - & - \\
\hline 27 & 3 & 9983 & 0 & 0 & 46513 & - & - \\
\hline 29 & 3 & 56454 & 0 & 0 & 42 & - & - \\
\hline 30 & 3 & 47180 & 8900 & 416 & 0 & - & - \\
\hline 31 & 3 & 56132 & 0 & 0 & 364 & - & - \\
\hline 33 & 3 & 53692 & 0 & 0 & 2804 & - & - \\
\hline 34 & 3 & 47600 & 0 & 0 & 8896 & - & - \\
\hline 35 & 3 & 41082 & 0 & 0 & 15414 & - & - \\
\hline 38 & 3 & 39550 & 0 & 0 & 16946 & - & - \\
\hline
\end{tabular}

This table illustrates relationship between ExPas and CoSets for S. cerevisiae metabolic network. The CoSets listed here correspond to those in Table 5. For each CoSet, we calculate how many ExPas cover $k$ reactions in it where $k$ ranges from 0 to size of this CoSet.

Since $a_{j}^{i}=0, j=k, \ldots, n$, then $\forall \mathbf{p}^{i} \in \mathbb{P}^{\prime \prime}, p_{j}^{i}=0, j=k, \ldots, n$, where $\mathbf{p}^{i}$ is the $i$ th ExPa in $\mathbb{P}$ and $p_{j}^{i}$ is the $j$ th component of $\mathbf{p}^{i}$.

Assuming that $\mathbb{P}^{\prime}=\left\{\mathbf{p}^{i} \mid \mathbf{p}^{i} \in \mathbb{P}\right.$ and $\left.p_{j}^{i}=0, j=k, \ldots, n\right\}$. Thus, $\mathbb{P}^{\prime \prime} \subseteq \mathbb{P}^{\prime}$.

Because $\mathbb{P}^{\prime} \subseteq \mathbb{A}$ and $\mathbb{P}^{\prime}$ is a systematically independent set, $\mathbb{P}^{\prime} \subseteq \mathbb{P}^{\prime \prime}$. Thus $\mathbb{P}^{\prime}=\mathbb{P}^{\prime \prime}$. Since the ExPa set of $G^{\prime}$ is unique, $\mathbb{P}^{\prime}$ is the ExPa set of $G^{\prime}$, and $\mathbb{P}^{\prime} \subseteq \mathbb{P}$.

\section{List of abbreviations used}

The abbreviations used in this study are listed in table 7.

\section{Competing interests}

The authors declare that they have no competing interests.

\section{Authors' contributions}

YX designed the system, implemented programs, carried out the analysis, and participated in manuscript prepara- tion. YPC supervised the project and suggested ways of improving the study, and participated in writing the manuscript. MC helped implement programs. WW participated in discussion of the research. FW designed and directed the research, and drafted the manuscript. All authors read and approved the final manuscript. 
Table 7: List of abbreviations used in this study.

\begin{tabular}{|c|c|c|c|}
\hline \multicolumn{4}{|c|}{ Concept Abbreviation } \\
\hline COBRA & Constraint-based reconstruction and analysis & EM & Elementary flux mode \\
\hline CoSet & Correlated reaction set & RBC & Human Red Blood Cell \\
\hline ExPa & Extreme pathway & & \\
\hline \multicolumn{4}{|c|}{ Metabolite Abbreviation } \\
\hline AKG & Alpha ketoglutarate & MAL & Malate \\
\hline GLC & Glucose & G6P & Glucose-6-phosphate \\
\hline F6P & Fructose-6-phosphate & FDP & Fructose-1,6-phosphate \\
\hline DHAP & Dihydroxyacetone phosphate & GA3P & Glyceraldehyde-3-phosphate \\
\hline I3DPG & I,3-Diphosphoglycerate & 23DPG & 2,3-Diphosphoglycerate \\
\hline $3 P G$ & 3-Phosphoglycerate & 2PG & 2-Phosphoglycerate \\
\hline PEP & Phosphoenolpyruvate & PYR & Pyruvate \\
\hline LAC & Lactate & 6PGL & 6-Phosphogluco-lactone \\
\hline 6PGC & 6-Phosphogluconate & RL5P & Ribulose-5-phosphate \\
\hline$\times 5 P$ & Xylulose-5-phosphate & R5P & Ribose-5-phosphate \\
\hline S7P & Sedoheptulose-7-phosphate & E4P & Erythrose-4-phosphate \\
\hline PRPP & 5-Phosphoribosyl-I-pyrophosphate & IMP & Inosine monophosphate \\
\hline RIP & Ribose-I-phosphate & $\mathrm{HX}$ & Hypoxanthine \\
\hline INO & Inosine & $\mathrm{ADE}$ & Adenine \\
\hline ADO & Adenosine & AMP & Adenosine monophosphate \\
\hline ADP & Adenosine diphosphate & ATP & Adenosine triphosphate \\
\hline NAD & Nicotinamide adenine dinucleotide & $\mathrm{H}$ & Hydrogen lon \\
\hline $\mathrm{NADH}$ & Nicotinamide adenine dinucleotide(R) & $\mathrm{NH} 3$ & Ammonia \\
\hline NADP & Nicotinamide adenine dinucleotide phosphate & $\mathrm{Pi}$ & Inorganic Phosphate \\
\hline NADPH & Nicotinamide adenine dinucleotide phosphate(R) & $\mathrm{CO} 2$ & Carbon Dioxide \\
\hline $\mathrm{H} 2 \mathrm{O}$ & Water & & \\
\hline
\end{tabular}

Pathway/Reaction Abbreviation

\begin{tabular}{|c|c|c|c|}
\hline EMP & Embden-Meyerhof-Parnas pathway & PPP & Pentose Phosphate Pathway \\
\hline $34 \mathrm{HPPt} 2 \mathrm{~m}$ & 34 hydroxyphenyl pyruvate mitochondrial transport via proton symport & $\mathrm{ACKr}$ & acetate kinase \\
\hline ACOTAim & acteylornithine transaminase irreversible mitochondrial & ACONT & aconitase \\
\hline $\mathrm{ACt} 2 \mathrm{r}$ & acetate reversible transport via proton symport & ABTA & 4 aminobutyrate transaminase \\
\hline AGPRim & $\mathrm{N}$ acetyl g glutamyl phosphate reductase irreversible mitochondrial & ACSm & acetyl CoA synthetase \\
\hline AKGDbm & oxoglutarate dehydrogenase dihydrolipoamide S succinyltransferase & ADHEr & Acetaldehyde dehydrogenase \\
\hline ACGKm & acetylglutamate kinase mitochondrial & ALD & Aldolase \\
\hline AKGDam & oxoglutarate dehydrogenase lipoamide & ADKIm & adenylate kinase mitochondrial \\
\hline AdPRT & Adenine phosphoribosyl transferase & ADKI & adenylate kinase \\
\hline AKGMAL & alpha ketoglutaratemalate transporter & AKGDH & 2 Oxogluterate dehydrogenase \\
\hline AKGt2r & 2 oxoglutarate reversible transport via symport & ARGSL & argininosuccinate lyase \\
\hline ARGSSr & argininosuccinate synthase reversible & ACLSm & acetolactate synthase mitochondrial \\
\hline CYOR_u6m & ubiquinol 6 cytochrome $\mathrm{c}$ reductase & CS & citrate synthase \\
\hline CYOOm & cytochrome $\mathrm{c}$ oxidase mitochondrial & $\mathrm{CO} 2 \mathrm{t}$ & $\mathrm{CO} 2$ transporter via diffusion \\
\hline CYTBD & cytochrome oxidase bd ubiquinol 82 protons & CYTK2 & cytidylate kinase dCMP \\
\hline D_LACt2 & D lactate transport via proton symport & DCTPD & dCTP deaminase \\
\hline DHADIm & dihydroxy acid dehydratase 23 dihydroxy 3 methylbutanoate mitochondrial & EN & Enolase \\
\hline ETOHt2r & ethanol reversible transport via proton symport & ENO & enolase \\
\hline EX_ac(e) & Acetate exchange & EX_ADE & ADE exchange \\
\hline EX_akg(e) & 2 Oxoglutarate exchange & EX_co2(e) & CO2 exchange \\
\hline EX_etoh(e) & Ethanol exchange & EX_for(e) & Formate exchange \\
\hline EX_fum(e) & Fumarate exchange & EX_glc(e) & D Glucose exchange \\
\hline EX_h2o(e) & $\mathrm{H} 2 \mathrm{O}$ exchange & EX_LAC & LAC exchange \\
\hline EX_lac_D(e) & D lactate exchange & EX_NAD & NAD exchange \\
\hline EX_NADH & NADH exchange & EX_NADP & NADP exchange \\
\hline EX_NADPH & NADPH exchange & EX_Pyr(e) & Pyruvate exchange \\
\hline FBA & fructose bisphosphate aldolase & EX_o2(e) & Ó2 exchange \\
\hline FORt & formate transport via diffusion & G6PDH2r & glucose 6 phosphate dehydrogenase \\
\hline GCCam & glycine cleavage complex lipoylprotein mitochondrial & GND & phosphogluconate dehydrogenase \\
\hline
\end{tabular}


Table 7: List of abbreviations used in this study. (Continued)

\begin{tabular}{|c|c|c|c|}
\hline $\mathrm{GCCcm}$ & glycine cleavage complex lipoylprotein mitochondrial & GLCpts & D glucose transport via PEPPyr PTS \\
\hline GCCbim & glycine cleavage complex lipoylprotein irreversible mitochondrial & GLUDC & Glutamate Decarboxylase \\
\hline GAPD & glyceraldehyde 3 phosphate dehydrogenase & HETZK & hydroxyethylthiazole kinase \\
\hline GAPDH & Glyceraldehyde phosphate dehydrogenase & $\mathrm{H} 2 \mathrm{Ot}$ & $\mathrm{H} 2 \mathrm{O}$ transport via diffusion \\
\hline HMPKI & hydroxymethylpyrimidine kinase ATP & $\mathrm{ICL}$ & Isocitrate lyase \\
\hline KARAIim & acetohydroxy acid isomeroreductase mitochondrial & LDH & Lactate dehydrogenase \\
\hline NDPK7 & nucleoside diphosphate kinase ATPdCDP & MALS & malate synthase \\
\hline MALt2r & $\mathrm{L}$ malate reversible transport via proton symport & LDH_D & D lactate dehydrogenase \\
\hline $\mathrm{O} 2 \mathrm{t}$ & o2 transport diffusion & $\mathrm{O} 2 \mathrm{tm}$ & O2 transport diffusion \\
\hline OCBTi & ornithine carbamoyltransferase irreversible & PFK & Phosphofructokinase \\
\hline ORNTACim & ornithine transacetylase irreversible mitochondrial & PGM & Phosphoglyceromutase \\
\hline ORNt3m & ornithine mitochondrial transport via proton antiport & PFL & Pyruvate formate lyase \\
\hline PGCD & phosphoglycerate dehydrogenase & PGI & Phosphoglucoisomerase \\
\hline PGK & phosphoglycerate kinase & PGL & 6 phosphogluconolactonase \\
\hline PGL & 6-phosphoglyconolactonase & PGM & phosphoglycerate mutase \\
\hline PDGH & 6-phosphoglycononate dehydrogenase & PMPK & phosphomethylpyrimidine kinase \\
\hline PGPPAm_SC & phosphatidylglycerol phosphate phosphatase A yeast specific mitochondrial & PK & Pyruvate kinase \\
\hline PNPase & Purine nucleoside phosphorylase & PPS & phosphoenolpyruvate synthase \\
\hline PRM & Phosphoribomutase & PSERT & phosphoserine transaminase \\
\hline PSP_L & phosphoserine phosphatase $L$ serine & PTAr & phosphotransacetylase \\
\hline PYRt2r & pyruvate reversible transport via proton symport & R5PI & Ribose-5-phosphate isomerase \\
\hline SSALy & succinate semialdehyde dehydrogenase NADP & SUCD4 & succinate dehyrdogenase \\
\hline SUCOAS & succinyl CoA synthetase ADP forming & TA & Transaldolase \\
\hline SUCOASm & Succinate CoA ligase ADP forming & TALA & transaldolase \\
\hline TYRt2m & tyrosine mitochondrial transport via proton symport & TKII & Transketolase \\
\hline TYRTAm & tyrosine transaminase mitochondrial & TKTI & transketolase \\
\hline TMPPP & thiamine phosphate diphosphorylase & TMN & thiaminase \\
\hline TPI & Triose phosphate isomerase & TKI & Transketolase \\
\hline Xu5PE & Xylulose-5-phosphate epimerase & & \\
\hline
\end{tabular}

Abbreviations used in this study are divided into three parts. They are concept abbreviations, metabolite abbreviations and pathway/reaction abbreviations.

\section{Additional material}

\section{Additional file 1}

The reaction abbreviation list of $E$. coli core metabolic network. This is an $\mathrm{Excel}^{\varpi}$ file of reaction abbreviations and reaction names of $\mathrm{E}$. coli core metabolic network.

Click here for file

[http://www.biomedcentral.com/content/supplementary/14712105-10-S1-S58-S1.xls]

\section{Additional file 2}

Maps of Reactions and ExPas of RBC metabolic network. This is a PDF file with a table and a figure. The table describes all the internal reactions in RBC metabolic network and the figure shows all the type I and II ExPas of this model.

Click here for file

[http://www.biomedcentral.com/content/supplementary/14712105-10-S1-S58-S2.pdf]

\section{Additional file 3}

The reaction abbreviation list of S. cerevisiae metabolic network. This is an Excel $^{\varpi}$ file of reaction abbreviations and reaction names of S. cerevisiae metabolic network.

Click here for file

[http://www.biomedcentral.com/content/supplementary/14712105-10-S1-S58-S3.xls]

\section{Additional file 4}

All the CoSets of S. cerevisiae metabolic network. This is an Excel $^{\circledR}$ file of all the 135 CoSets of S. cerevisiae metabolic network.

Click here for file

[http://www.biomedcentral.com/content/supplementary/14712105-10-S1-S58-S4.xls]

\section{Additional file 5}

Relationship between ExPas and CoSets for S. cerevisiae model (full version). This is an Excel $^{\varpi}$ file of relationship between ExPas and all the 135. CoSets for $\mathrm{S}$. cerevisiae model.

Click here for file

[http://www.biomedcentral.com/content/supplementary/1471-

2105-10-S1-S58-S5.xls]

\section{Acknowledgements}

This work is supported by grants 60673016,60496324 of Chinese National Natural Science Foundation and 863 project (No. 2006AA02Z324).

This article has been published as part of BMC Bioinformatics Volume I0 Supplement I, 2009: Proceedings of The Seventh Asia Pacific Bioinformatics Conference (APBC) 2009. The full contents of the supplement are available online at http://www.biomedcentral.com/I47|-2/05/I0? issue=SI 


\section{References}

I. Reed JL, Famili I, Thiele I, Palsson BO: Towards multidimensional genome annotation. Nat Rev Genet 2006, 7(2): |30-|4|.

2. Covert MW, Schilling CH, Famili I, Edwards JS, Goryanin II, Selkov E, Palsson BO: Metabolic modeling of microbial strains in silico. Trends Biochem Sci 200I, 26(3): I79-186.

3. Edwards JS, Covert M, Palsson BO: Metabolic modelling of microbes: the flux-balance approach. Environ Microbiol 2002 , 4(3): I 33-40

4. Reed JL, Palsson BO: Thirteen years of building constraintbased in silico models of Escherichia coli. | Bacteriol 2003, I 85(9):2692-2699.

5. Price ND, Reed JL, Palsson BO: Genome-scale models of microbial cells: evaluating the consequences of constraints. Nat Rev Microbiol 2004, 2(I I):886-97.

6. Covert MW, Palsson BO: Constraints-based models: regulation of gene expression reduces the steady-state solution space. J Theor Biol 2003, 22 I(3):309-325.

7. Schilling $\mathrm{CH}$, Edwards JS, Letscher D, Palsson BO: Combining pathway analysis with flux balance analysis for the comprehensive study of metabolic systems. Biotechnol Bioeng 2000 , 7 I(4):286-306.

8. Schilling $\mathrm{CH}$, Letscher $\mathrm{D}$, Palsson $\mathrm{BO}$ : Theory for the systemic definition of metabolic pathways and their use in interpreting metabolic function from a pathway-oriented perspective. J Theor Biol 2000, 203(3):229-248.

9. Schilling $\mathrm{CH}$, Palsson $\mathrm{BO}$ : The underlying pathway structure of biochemical reaction networks. Proc Natl Acad Sci USA 1998, 95(8):4193-4198.

10. Papin JA, Stelling J, Price ND, Klamt S, Schuster S, Palsson BO: Comparison of network-based pathway analysis methods. Trends Biotechnol 2004, 22(8):400-405.

II. Price ND, Reed JL, Papin JA, Famili I, Palsson BO: Analysis of metabolic capabilities using singular value decomposition of extreme pathway matrices. Biophys / 2003, 84(2 Pt I ):794-804.

12. Schuster S, Fell DA, Dandekar T: A general definition of metabolic pathways useful for systematic organization and analysis of complex metabolic networks. Nat Biotechnol 2000 I 8(3):326-32

13. Schuster S, Dandekar T, Fell DA: Detection of elementary flux modes in biochemical networks: a promising tool for pathway analysis and metabolic engineering. Trends Biotechnol 1999, I 7(2):53-60.

14. Schuster S, Hilgetag C: On elementary flux modes in biochemical reaction systems at steady state. Journal of Biological Systems (JBS) 1994, 2(2): 165-182.

15. Klamt S, Stelling J: Two approaches for metabolic pathway analysis? Trends Biotechnol 2003, 2 I(2):64-9.

16. Papin JA, Reed JL, Palsson BO: Hierarchical thinking in network biology: the unbiased modularization of biochemical networks. Trends Biochem Sci 2004, 29( I 2):64I-647.

17. Stelling J, Klamt S, Bettenbrock K, Schuster S, Gilles ED: Metabolic network structure determines key aspects of functionality and regulation. Nature 2002, 420(69 I 2): 190-3.

18. Price ND, Papin JA, Palsson BO: Determination of redundancy and systems properties of the metabolic network of Helicobacter pylori using genome-scale extreme pathway analysis. Genome Res 2002, I 2(5):760-769.

19. Klamt S, Stelling J: Combinatorial complexity of pathway analysis in metabolic networks. Mol Biol Rep 2002, 29(I-2):233-6.

20. Yeung $M$, Thiele I, Palsson BO: Estimation of the number of extreme pathways for metabolic networks. BMC Bioinformatics 2007, 8:363.

21. Almaas E, Kovacs B, Vicsek T, Oltvai ZN, Barabasi AL: Global organization of metabolic fluxes in the bacterium Escherichia coli. Nature 2004, 427(6977):839-43.

22. Becker SA, Feist AM, Mo ML, Hannum G, Palsson BO, Herrgard MJ: Quantitative prediction of cellular metabolism with constraint-based models: the COBRA Toolbox. Nat Protoc 2007, 2(3):727-738.

23. Sarlyar B, Perk S, Akman U, Hortacsu A: Monte Carlo sampling and principal component analysis of flux distributions yield topological and modular information on metabolic networks. Journal of Theoretical Biology 2006, 242(2):389-400.

24. Reed JL, Palsson BO: Genome-scale in silico models of E. coli have multiple equivalent phenotypic states: assessment of correlated reaction subsets that comprise network states. Genome Res 2004, I 4(9): I797-I805

25. Wiback SJ, Palsson BO: Extreme Pathway Analysis of Human Red Blood Cell Metabolism. Biophys J 2002, 83(2):808-8I8.

26. In Silico Organisms, E. coli, E. coli SBML [http://gcrg.ucsd.edu/ In Silico Organisms/E coli/E coli SBML]

27. Palsson BO: Systems Biology: Properties of Reconstructed Networks Ist edition. Cambridge University Press; 2006.

28. Mulquiney PJ, Kuchel PW: Model of 2,3-bisphosphoglycerate metabolism in the human erythrocyte based on detailed enzyme kinetic equations: computer simulation and metabolic control analysis. Biochem J I999, 342(Pt 3):597-604.

29. Lee ID, Palsson BO: A comprehensive model of human erythrocyte metabolism: extensions to include $\mathrm{pH}$ effects. Biomed Biochim Acta 1990, 49(8-9):771-789.

30. Joshi A, Palsson BO: Metabolic dynamics in the human red cell. Part I-A comprehensive kinetic model. J Theor Biol 1989 | 4 | (4):5| 5-528.

31. Joshi A, Palsson BO: Metabolic dynamics in the human red cell. Part II-Interactions with the environment. J Theor Biol 1989 , I 4 I (4):529-545.

32. Nelson DL, Cox MM: Lehninger Principles of Biochemistry 4th edition. New York: W. H. Freeman and Company; 2005.

33. Duarte NC, Herrgard MJ, Palsson BO: Reconstruction and validation of Saccharomyces cerevisiae iND750, a fully compartmentalized genome-scale metabolic model. Genome Res 2004, I 4(7): | 298-309.

34. Bell SL, Palsson BO: expa: a program for calculating extreme pathways in biochemical reaction networks. Bioinformatics 2005, 2 I (8): I739-1740

35. Schilling C, Schuster S, Palsson B, Heinrich R: Metabolic Pathway Analysis: Basic Concepts and Scientific Applications in the Post-genomic Era. Biotechnology Progress I999, I 5(3):296-303.

36. Price ND, Famili I, Beard DA, Palsson BO: Extreme Pathways and Kirchhoff's Second Law. Biophys J 2002, 83(5):2879-2882.
Publish with Bio Med Central and every scientist can read your work free of charge

"BioMed Central will be the most significant development for disseminating the results of biomedical research in our lifetime. "

Sir Paul Nurse, Cancer Research UK

Your research papers will be:

- available free of charge to the entire biomedical community

- peer reviewed and published immediately upon acceptance

- cited in PubMed and archived on PubMed Central

- yours - you keep the copyright
BioMedcentral 